\title{
Inclusion Bodies in Tick-Borne Diseases Diagnosed in Patients from Northern Wisconsin
}

\author{
Mrinal M. Patnaik, MD
}

\section{Clinical Vignette}

Case 1

A 65-year-old man from northern Wisconsin, presented to the clinic with complaints of fever, chills, myalgias and severe fatigue. He lived on a farm and frequently visited the woods for hiking. On examination he was found to be febrile without hepatosplenomegaly or lymphadenopathy. His hemoglobin was $8 \mathrm{~g} / \mathrm{dL}$, white count was 2.8 with 1100 neutrophils, and the platelet count was $55,000 / \mathrm{mm}^{3}$. A peripheral smear was ordered.

Examination of the Wright-stained peripheral smear (figure 1) shows a neutrophil with an intracytoplasmic inclusion body termed a morulae (Latin for mulberry). The presence of such intra-cytoplasmic inclusions in neutrophils is classically associated with human granulocytic anaplasmosis. The patient was successfully treated with doxycycline $100 \mathrm{mg}$ orally twice a day for 10 days. On a follow up visit he was found to be asymptomatic with complete normalization of his blood counts.

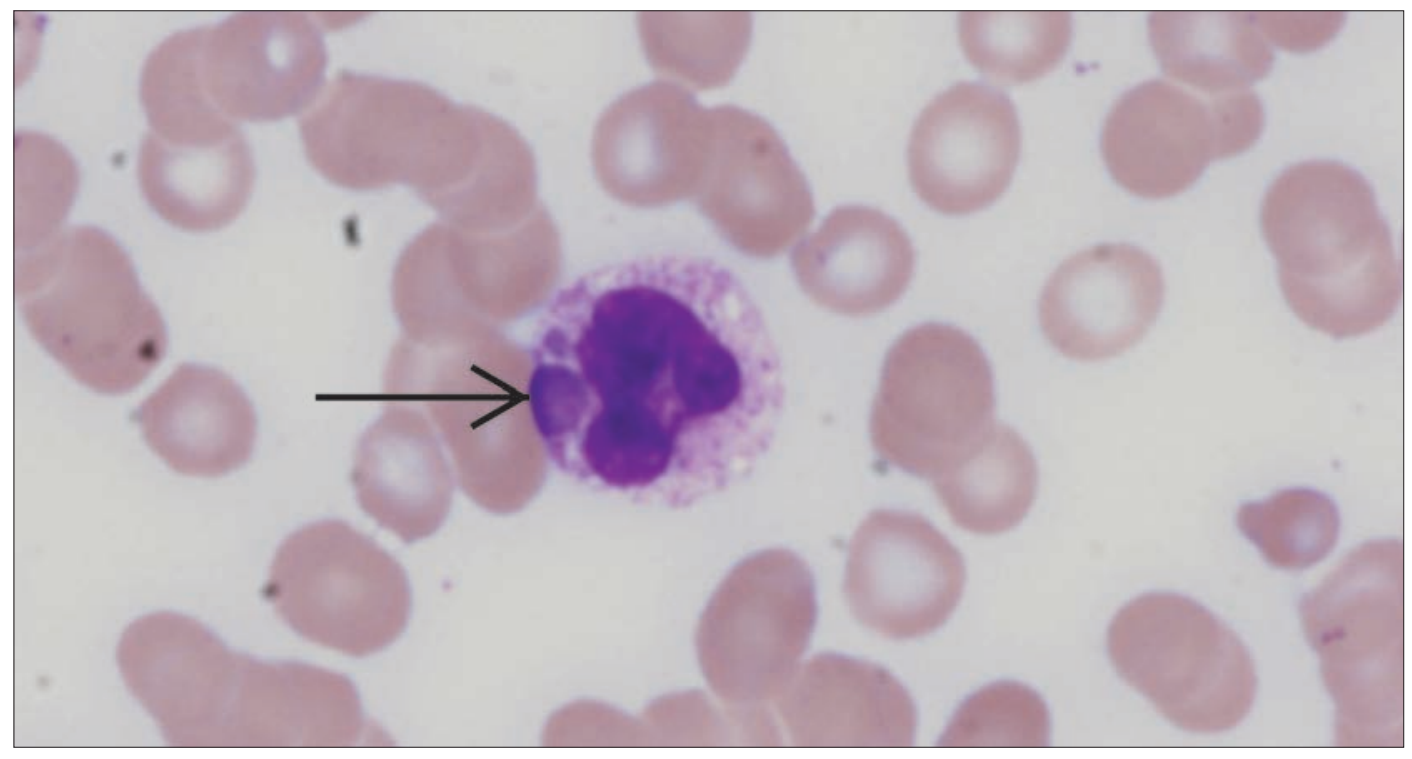

Figure 1. Wright-stained peripheral smear showing a neutrophil with an intracytoplasmic inclusion body, or morulae (arrow), classically associated with human granulocytic anaplasmosis.

Corresponding Author

Mrinal M. Patnaik, MD

Department of Internal Medicine

University of Minnesota

Minneapolis, MN 55455

Tel: (6I2) 720-9150

Email:mrinal.patnaik@gmail.com
Keywords: Fever, Peripheral smear, Maltese cross, Morulae

Received: May 30, 2008

Ist Revision: June 27, 2008

2nd Revision: September 25, 2008

Accepted: September 26, 2008

doi: $10.312 \mid / \mathrm{cmr} .2009 .807 .818$

The Aperture, like the opening in the lens of a microscope that allows light to pass through, is a forum for art, humor, and images that provides a portal for new or different views of medicine and research. 




Figure 2. Peripheral smear showing red blood cells with parasitic inclusions forming a piroplasmic tetrad, or maltese cross (arrow), pathognomonic for babesiosis.

\section{Case 2}

A 35-year-old man from northern Wisconsin, presented to the clinic with complaints of fever, chills and severe fatigue. He lived on a farm and frequently visited the woods for hiking. On examination he was found to be febrile with presence of a mild splenomegaly. His hemoglobin was $8 \mathrm{~g} / \mathrm{dL}$ and the platelet count was $35,000 / \mathrm{mm}^{3}$. A peripheral smear was ordered.

Red blood cells with parasitic inclusions were seen in the patient's peripheral smear (figure 2). The parasites are 1 to $5 \mu \mathrm{m}$ in diameter with spherical, oval or tear drop shapes. The pathognomonic feature is the presence of the parasitic piroplasmic tetrad in the red cells, forming the Maltese cross. This smear is diagnostic for infection with Babesia microti in humans. The patient was successfully treated with clindamycin and quinine.

\section{Discussion}

The term "Human Ehrlichiosis" describes a spectrum of related tick borne infections in human beings. The three most commonly seen infections are human granulocytic anaplasmosis (HGA) caused by Anaplasma phagocytophilum, human monocytic ehrlichiosis (HME) caused by Ehrlichia chaffeensis, and human ewingii ehrlichiosis caused by Ehrlichia ewingii. ${ }^{1}$ The commonly associated vectors include Ixodes scapularis in the eastern United States, and Ixodes pacificus in the western United States, with small mammals such as white-footed mice being likely reservoirs. ${ }^{2}$

Blood smear evaluation is useful in the diagnosis of HGA. In the U.S., between $25 \%$ to $75 \%$ of cases reported demonstrate morulae in the peripheral blood examination, with the maximal sensitivity of this modality being during the first week of infection. ${ }^{3}$ Although no clinical trials have been conducted, current data suggest that all forms of ehrlichiosis respond well to tetracyclines. The currently recommended treatment regimens for HGA and HME include doxycycline $100 \mathrm{mg}$ orally or intravenously every 12 hours for 5 to 14 days, or rifampin $300 \mathrm{mg}$ orally every 12 hours for 7 to 10 days. ${ }^{4,5}$

The Maltese cross formation, pathognomonic for the diagnosis of babesiosis, is often described in infections with Babesia microti, Babesia equi and Theileria parva. In mice infected with Babesia microti, Maltese cross formations have been thought to represent a protective response against the host immune system, creating an environment favorable for the progression of the parasitemia. ${ }^{6}$ This phenomenon, however, has not yet been described in human beings, and remains a theoretical concept yet to be confirmed. ${ }^{7,8}$

The combination of quinine and clindamycin has long been considered standard therapy for the treatment of babesiosis. ${ }^{5}$ However, this combination has been associated with a relatively high incidence of adverse drug reactions such as nausea, vomiting, tinnitus, diarrhea and cardiac dysrhythmia. ${ }^{5}$ In a prospective, non-blinded, randomized trial, a combination of azithromycin (500 $\mathrm{mg}$ on day one, followed by $250 \mathrm{mg}$ for 6 days) and atovaquone ( $750 \mathrm{mg}$ every 12 hours for 7 days) was found to be as effective as standard therapy with quinine and clindamycin, for the treatment of non-life threatening babesiosis, and more importantly, was associated with fewer adverse effects. ${ }^{9}$

\section{References}

1. Dumler JS, Madigan JE, Pusterla N, Bakken JS. Ehrlichioses in humans: epidemiology, clinical presentation, diagnosis, and treatment. Clin Infect Dis 2007;45 (Suppl 1):S45-51.

2. Dumler JS. Anaplasma and Ehrlichia infection. Ann N Y Acad Sci 2005;1063:361-373. 
3. Bakken JS, Aguero-Rosenfeld ME, Tilden RL, Wormser GP, Horowitz HW, Raffalli JT, Baluch M, Riddell D, Walls JJ, Dumler JS. Serial measurements of hematologic counts during the active phase of human granulocytic ehrlichiosis. Clin Infect Dis 2001;32:862-870.

4. Maurin M, Bakken JS, Dumler JS. Antibiotic susceptibilities of Anaplasma (Ehrlichia) phagocytophilum strains from various geographic areas in the United States. Antimicrob Agents Chemother 2003;47:413-415.

5. Wormser GP, Dattwyler RJ, Shapiro ED, Halperin JJ, Steere AC, Klempner MS, Krause PJ, Bakken JS, Strle F, Stanek G, Bockenstedt L, Fish D, Dumler JS, Nadelman RB. The clinical assessment, treatment, and prevention of lyme disease, human granulocytic anaplasmosis, and babesiosis: clinical practice guidelines by the Infectious Diseases Society of America. Clin Infect Dis 2006;43:1089-1134.

6. Yokoyama N, Bork S, Nishisaka M, Hirata H, Matsuo T, Inoue N, Xuan X, Suzuki H, Sugimoto C, Igarashi I. Roles of the Maltese cross form in the development of parasitemia and protection against Babesia microti infection in mice. Infect Immun 2003;71:411-417.

7. Kjemtrup AM, Conrad PA. Human babesiosis: an emerging tick-borne disease. Int J Parasitol 2000;30:1323-1337.

8. Camacho T. Roles of the Maltese cross form of Babesia microti in the development of parasitemia in B. microti infection. Infect Immun 2004;72:4929.

9. Krause PJ, Lepore T, Sikand VK, Gadbaw J Jr, Burke G, Telford SR 3rd, Brassard P, Pearl D, Azlanzadeh J, Christianson D, McGrath D, Spielman A. Atovaquone and azithromycin for the treatment of babesiosis. N Engl J Med 2000; 343:1454-1458. 